\title{
Posture prediction and optimization for a manual assembly operation involving lifting of weights
}

\author{
Biswaranjan Rout ${ }^{1, *}$, Rati Ranjan Dash ${ }^{2}$, and Debabrata Dhupal ${ }^{1}$ \\ ${ }^{1}$ Department of Production Engineering, VSSUT, Burla 768018, Odisha, India \\ 2 Department of Mechanical Engineering, CET, Bhubaneswar 751003, Odisha, India
}

Received: 26 January 2019 / Accepted: 2 December 2019

\begin{abstract}
The present work combines ergonomics with the posture prediction in the assembly process to avoid musculoskeletal issues of human operator. For improved productivity the operator should be in a better work environment and in sound health. The purpose of this paper is to provide a different perspective to avoid ergonomic risk factors in manual assembly. Here, a human is modeled as 20-DOF as modeled in robotic analysis and simulated in a virtual environment. In the present study, two objective cost functions i.e. joint discomfort function and energy expenditure function have been employed for evaluating the optimized posture. For posture prediction, a combined multi-objective optimization (MOO) method is used and the objective cost functions are minimized i.e. less joint discomfort and less energy in MOO method required to do the manual assembly operation and consequently, the results are compared and finally the movements are tested using REBA technique.
\end{abstract}

Keywords: Ergonomics / posture prediction / joint discomfort function / energy function / MOO / REBA

\section{Introduction}

Precise simulation of assembly posture is necessary of the posture prediction for ergonomic assessment. However, manual manipulation of human models is required by the ergonomics simulation tools, which results in errors and less efficiency. The accuracy of the posture prediction method in non-automated and semi automated workplace should satisfy the ergonomic condition. Most importantly, this method helps in providing a better understanding in predicting realistic postures for varied assembly conditions for the ergonomic analysis of the human performance.

This work proposes development of a generalized posture prediction method in assembly job simulation for the manual assembly tasks. At first, a human model is proposed, where the human body represents a system of kinematic chain with a number of revolute joints connecting the series of links. The model is same as in robotics; the open chain mechanism is used for analysis and application which are connected by joints serially to the links [1]. For finding a suitable posture prediction procedure, a human's anthropometric characteristic, for example, masses of body segments, body dimensions, are taken into consideration. We have also taken some assumptions to simulate the

\footnotetext{
* e-mail: biswa.pp@gmail.com
}

human body: they are flexion and extensions of the neck; rotational movement of trunk is not considered [2]. The discomfort and energy expenditure in joints are to be less in order to avoid musculoskeletal problems in human operators. In present work we have considered the human for posture prediction in different path in lifting weight in a packaging industry.

For the model to achieve the optimized posture prediction the use of multi objective optimization (MOO) technique used to find the effectiveness of the algorithm using multiple objectives in assembly task is described. In this work we have taken two objective functions that are energy expenditure function and joint discomfort function. The former deals with human postures to optimize effort of the job and the later deals with the survival capacity to predict fatigue of the body.

The joint limitations in human body parts, the work space limitations and reference point for the human are the constraints considered for optimization problem. The basic constrain taken for optimization of posture prediction problems is a hand that will be in contact with the targeted object. Also, we have considered different paths for lifting the weight.

Weight of the object, working distance, joint limitations, posture analysis and clearance analysis visibility, assembly force and clearance, are the common ergonomic requirements of a manual industry. In vision analysis parts, 

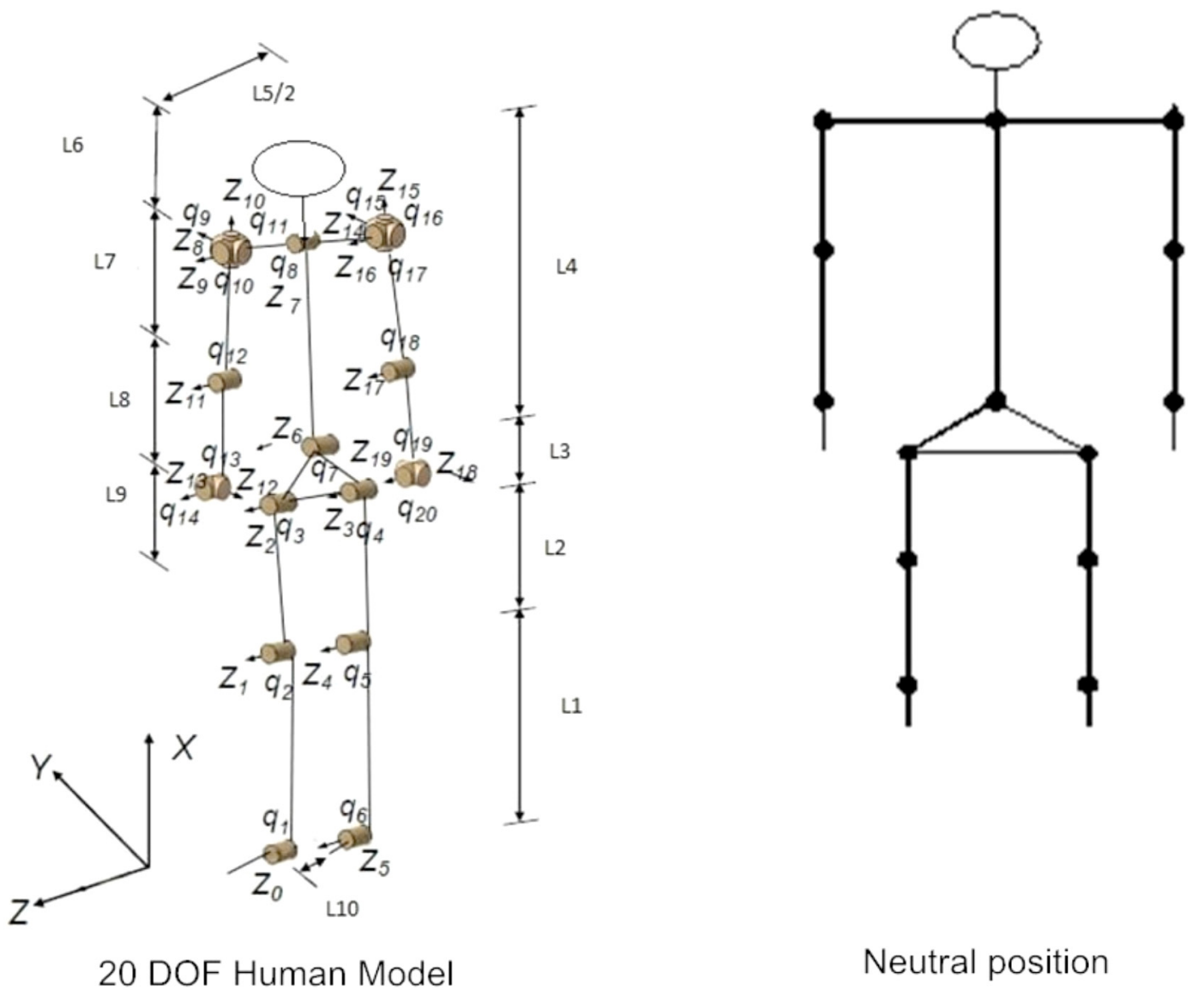

Neutral position

Fig. 1. Showing the human model with neutral position.

or components or operations must be able to see when assembled. In posture analysis weight of the object, working distance and assembly force are calculated. If weight limits are exceeded, a weight reduction of the part must be made or an adequate lifting device be developed.

Various researchers have employed methods which include experimental, statistical, and analytical approach in posture prediction of human with various degrees of freedom [3-13] for modeling. Inverse dynamics [3] and image processing tools [4] are also used by various researchers to predict posture and optimization using GA [9-11], ANN [15], MOO [16-23] to attain the posture prediction of human.

Rapid Entire Body Assessment (REBA) is ergonomic tool for posture analysis. REBA is developed using postural analysis and musculoskeletal risk factor in variety task with a quantitative indicator, calculating the average of each involved muscle activities in joints. In this work the optimized posture is validated using REBA [31].

From the literature reviews it is observed that, yet almost no study concerning 20-DOF has been reported in posture prediction of human that would ensure scope for researchers. Novelty aspect, the present study focuses on a 20-DOF human model for posture prediction. The objective cost functions taken are joint discomfort function and energy expenditure rate. MOO is used as optimization method to predict posture in assembly operation where different weights are to be lifted.

\section{Human modeling}

Human body consists of foot, leg, spine, torso, head, hand and wrist joined by various joints which have relative motion when humans do some work similar to the links of the robotic manipulator system. Depending upon DOF of joints and weight of the individual body parts each can be modeled as the link and joint of robotic arm [14].

In this work we have taken as 20 degrees of freedom human model for posture prediction as in our previous work. In the musculoskeletal system of human, different joints are considered as revolute joints joining the links or bones which are considered as rigid bodies. Different joints considered are ankle joint, knee joint, hip joint, trunk joint, shoulder joint, elbow joint and wrist joints. Out of which shoulder joint is modeled as $3 \mathrm{DOF}$, wrist is modeled as 2 DOF and other joints are 1 DOF each. In Figure 1 human model and the most neutral position is shown. In this work the movement of trunk and spine is not considered.

In this case the lengths of various joints for two subjects are: $\mathrm{L}_{1}$ is taken $460 \mathrm{~mm}$ and $400 \mathrm{~mm}, \mathrm{~L}_{2}$ is $400 \mathrm{~mm}$ and $380 \mathrm{~mm}, \mathrm{~L}_{3}$ is $140 \mathrm{~mm}$ and $120 \mathrm{~mm}, \mathrm{~L}_{4}$ is $680 \mathrm{~mm}$ and 
Table 1. DH parameter and limiting position for 20 DOF model.

\begin{tabular}{|c|c|c|c|c|c|c|c|}
\hline \multicolumn{5}{|c|}{ D-H Parameters } & \multicolumn{3}{|c|}{ Limiting position } \\
\hline Joints DOF & $\theta_{i}$ & $d_{i}$ & $\alpha_{i}$ & $a_{i}$ & Neutral position & Lower limit & Upper limit \\
\hline 1 & $-q_{1}$ & 0 & 0 & $\mathrm{~L}_{1}$ & 0 & -50 & 38 \\
\hline 2 & $\mathrm{q}_{2}$ & 0 & 0 & $\mathrm{~L}_{2}$ & 0 & 0 & 135 \\
\hline 3 & $\mathrm{q}_{3}$ & 0 & $-\pi$ & 0 & 0 & -18 & 113 \\
\hline 4 & $-\mathrm{q}_{4}$ & 0 & 0 & $\mathrm{~L}_{1}$ & 0 & -18 & 113 \\
\hline 5 & $\mathrm{q}_{5}$ & 0 & 0 & $\mathrm{~L}_{2}$ & 0 & 0 & 135 \\
\hline 6 & $\mathrm{q}_{6}$ & 0 & $-\pi$ & 0 & 0 & -50 & 38 \\
\hline 7 & $\mathrm{q}_{7}$ & 0 & 0 & $\mathrm{~L}_{3}$ & 0 & -19 & 56 \\
\hline 8 & $\mathrm{q}_{8}$ & 0 & 0 & 0 & 0 & -30 & 40 \\
\hline 9 & $\mathrm{q}_{9}$ & 0 & $\pi / 2$ & 0 & 0 & -60 & 170 \\
\hline 10 & $-q_{10}$ & 0 & $\pi / 2$ & 0 & 0 & -18 & 80 \\
\hline 11 & $\mathrm{q}_{11}$ & 0 & $\pi / 2$ & $-\mathrm{L} 7$ & 0 & -20 & 97 \\
\hline 12 & $-q_{12}$ & 0 & $\pi / 2$ & $-\mathrm{L}_{8}$ & 0 & 0 & 140 \\
\hline 13 & $-\mathrm{q}_{13}$ & 0 & $\pi / 2$ & 0 & 0 & -70 & 80 \\
\hline 14 & $\mathrm{q}_{14}$ & 0 & $\pi / 2$ & $-\mathrm{L}_{9}$ & 0 & -30 & 20 \\
\hline 15 & $\mathrm{q}_{15}$ & 0 & $\pi / 2$ & 0 & 0 & -60 & 170 \\
\hline 16 & $-q_{16}$ & 0 & $\pi / 2$ & 0 & 0 & -18 & 80 \\
\hline 17 & $\mathrm{q}_{17}$ & 0 & $\pi / 2$ & $-\mathrm{L} 7$ & 0 & -20 & 97 \\
\hline 18 & $-\mathrm{q}_{18}$ & 0 & $\pi / 2$ & $-\mathrm{L}_{8}$ & 0 & 0 & 140 \\
\hline 19 & $-\mathrm{q}_{19}$ & 0 & $\pi / 2$ & 0 & 0 & -70 & 80 \\
\hline 20 & $\mathrm{q}_{20}$ & 0 & $\pi / 2$ & $-\mathrm{L}_{9}$ & 0 & -30 & 20 \\
\hline
\end{tabular}

$580 \mathrm{~mm} \mathrm{~L}_{5}$ is $360 \mathrm{~mm}$ and $320 \mathrm{~mm}, \mathrm{~L}_{6}$ is $190 \mathrm{~mm}$ and $150 \mathrm{~mm}, \mathrm{~L}_{7}$ is $340 \mathrm{~mm}$ and $300 \mathrm{~mm}, \mathrm{~L}_{8}$ is $310 \mathrm{~mm}$ and $260 \mathrm{~mm}, \mathrm{~L}_{9}$ is $190 \mathrm{~mm}$ each, $\mathrm{L}_{10}$ is $150 \mathrm{~mm}$ and $140 \mathrm{~mm}$, respectively.

$\mathrm{DH}$ method is a proven method for mathematical modeling in the field of robotics. This mathematical modeling (DH method) is used for transformation of adjacent joint position with respect to the link. In this method various factors used are two link factors i.e. $\theta_{i}$ (link twist angle) and $d_{i}$ (link distance) and two joint factors $a_{i}$ (joint distance) and $\alpha_{i}$ (joint angle) to explain the position and orientation. In human biomechanics this DH method is used as in robotics assuming the body parts as link to be connected by joints. The standard $\mathrm{DH}$ algorithm is available in literature [24].

Neutral position is the most comfortable position of human and each joint is having a limiting value i.e. lower limit and upper limit. DH parameters and joint limitations are for the human model as shown in Table 1.

With the help of above parameters describe in Table 1 the position of different joints and relative angle between each body parts under consideration can be calculated which gives the posture prediction keeping the foot at fixed point and tip of the hand as the target point. The position of joint are depends upon the objective functions considered. The model is developed keeping the foot position unchanged, and changing the other joints to lift the job from its required position. The change in joint parameter is such that discomfort at the joint is less and with less energy expenditure.

\section{Posture prediction}

In this work a 20 DOF model is used to predict the posture of human operator for a lifting of weight in a manual assembly work. For this we have taken two objective cost functions, they are joint discomfort function and energy expenditure function.

\subsection{Joint discomfort function}

Discomfort function generally measures the difference of CG to the desired position with respect to the neutral position. In Table 1 each joint has a lower limitation and upper limitation. The discomfort value is small when it is at neutral position, if the joints are moving from the neutral position to the limiting value, the discomfort level increases. The basic aim is to reduce this discomfort level.

It is found that the joint discomfort function is minimum in its neutral position where no movement is there and is higher when going towards the upper limit of the joint and lower limit of the joint. QU and QL are especially designed provisions for the upper limit and the lower limit of each joint. The neutral position of the digital human is given in Figure 1. The values at neutral positions 
Table 2. Joint torque limits.

\begin{tabular}{lll}
\hline Joint & Torque (UL) in N.m & Torque (LL) in N.m \\
\hline Ankle & 100 & -100 \\
Knee & 100 & -100 \\
Hip & 100 & -100 \\
Trunk & 100 & -100 \\
Shoulder & 60 & -50 \\
Elbow & 50 & -50 \\
Wrist & 10 & -0 \\
\hline
\end{tabular}

and at the upper/lower limits of each joint are specified in Table 1. QU and QL are defined mathematically and discomfort function are derived $[25,26]$.

$$
\begin{aligned}
Q U_{i} & =\left(0.5 \sin \left(\frac{5.0\left(q_{i}^{U}-q_{i}\right)}{q_{i}^{U}-q_{i}^{L}}+1.571\right)+1\right)^{100}, \\
Q L_{i} & =\left(0.5 \sin \left(\frac{5.0\left(q_{i}-q_{i}^{L}\right)}{q_{i}^{U}-q_{i}^{L}}+1.571\right)+1\right)^{100}, \\
f_{\text {discomfort }} & =\frac{1}{G} q_{i}^{\text {norm }}=\frac{q_{i}-q_{i}^{N}}{q_{i}^{U}-q_{i}^{L}}, \\
& =\sum_{i=1}^{D O F}\left[\gamma_{i}\left(\Delta q_{i}^{\text {norm }}\right)^{2}+G * Q U_{i}+G * Q L_{i}\right] .
\end{aligned}
$$

\subsection{Energy function}

The total rate of energy expenditure $(E)$, which is expressed in Watts per kilogram, is defined as follows:

$$
\dot{E}=\dot{E}_{W}+\dot{E}_{B}
$$

where $E_{W}=$ mechanical power of muscle, $E_{B}=$ basal metabolic rate (BMR).

Normally, in the joint space only the force is considered for energy expenditure in human model. Following Kim et al. [28], the definition of mechanical power is given as dot product of joint torque $\left(\boldsymbol{\tau}_{i}\right)$ and joint velocity $\left(q_{i}\right)$. The total mechanical power $E_{M}$ is as follows:

$$
E_{W}=\sum_{i=1}^{D O F}\left|\tau_{i} q_{i}\right| .
$$

In Table 2, joint torque limits are given.

The BMR i.e. basal metabolic rate is the metabolic rate of a human being at rest [27]. It means the minimum quantity of energy necessary for functioning of human, without doing any kind of external work. Hase et al. [29] has presented the following BMR model:

$$
E_{B}=0.685 B W+29.8
$$

where BW is the body weight (kilogram).

For optimization of posture prediction, the method is used, which determines different position of joints with respect to reference point such that the objective cost function is minimum. This can be expressed mathematically as

$$
\begin{array}{ll}
\text { find: } & q \in R^{D O F} \\
\text { To minimize: } & f(q)=\left[f_{1}(q) f_{2}(q) \ldots f_{k}(q)\right]^{T} \\
\text { Subject to: } & q_{i}(q) \leq 0 i=1,2, \ldots . ., m, \\
h_{j}(q)=o j=1.2, \ldots ., e
\end{array}
$$

where $k$, Total number of objective functions, $m$, Total number of inequality constraints, $e$, Total number of equality constrains, $q \varepsilon E^{\mathrm{DOF}}=$ vector of design variables.

The optimal posture can be obtained by solving the optimization condition. For this case the problem can be defined as:

$$
\begin{array}{ll}
\text { find: } & q \in R^{20} \\
\text { To minimize: } & \text { Cost functions } \\
\text { Subject to: } & \| P(\text { hand })-P(\text { object }) \| \\
\leq \in, q_{i}^{L} \leq q_{i} \leq q_{i}^{U}, i=1,2, \ldots, 3
\end{array}
$$

In this work, cost functions refer to joint discomfort function and energy function. $q i^{\mathrm{U}}$ and $q i^{\mathrm{L}}$ define the upper and lower limits for $q i$ joint in that order. Limits are given for not to assume an unrealistic posture [24].

Multi Objective Optimization (MOO) method is a multi criteria decision-making process. MOO is used where more than one objective function are there. It is a welldeveloped method for posture prediction. Here we have taken two objective functions: joint discomfort function and energy expenditure function. For MOO method for posture prediction is redefined as [30]:

$$
\begin{array}{cl}
\text { To minimize: } & f=w_{1} f_{1}^{N}+w_{2} f_{2}^{N} \\
& \| P(\text { hand }-P(\text { object })) \| \leq \in \\
\text { Subject to: } & q_{i}^{L} \leq q_{i} \leq q_{i}^{U} i=1, \ldots ., 20 \\
& \sin \left(q_{2}^{U}-q_{3}^{U}\right) L_{1} \leq X_{\text {hip }} \leq\left(L_{1}+L_{2}\right)
\end{array}
$$

where, $f_{1} N$ is the joint discomfort function and $f_{2} N$ is the energy function. $\varepsilon$ is the positive infinitesimal value which is equal to zero. The $\mathrm{P}$ (hip) can be found out, joint movement and anthropometric data. Weight factors $w_{1}$ and $w_{2}$ are given to discomfort function and energy expenditure function, respectively.

For optimization, MATLAB ${ }^{\circledR}$ optimization toolbox is used. The function "fmincon" is used to find the minimum discomfort function and energy expenditure function. It finds the constrained minimum of a scalar function of different variables from initial point to target point. It uses SQP algorithm. 


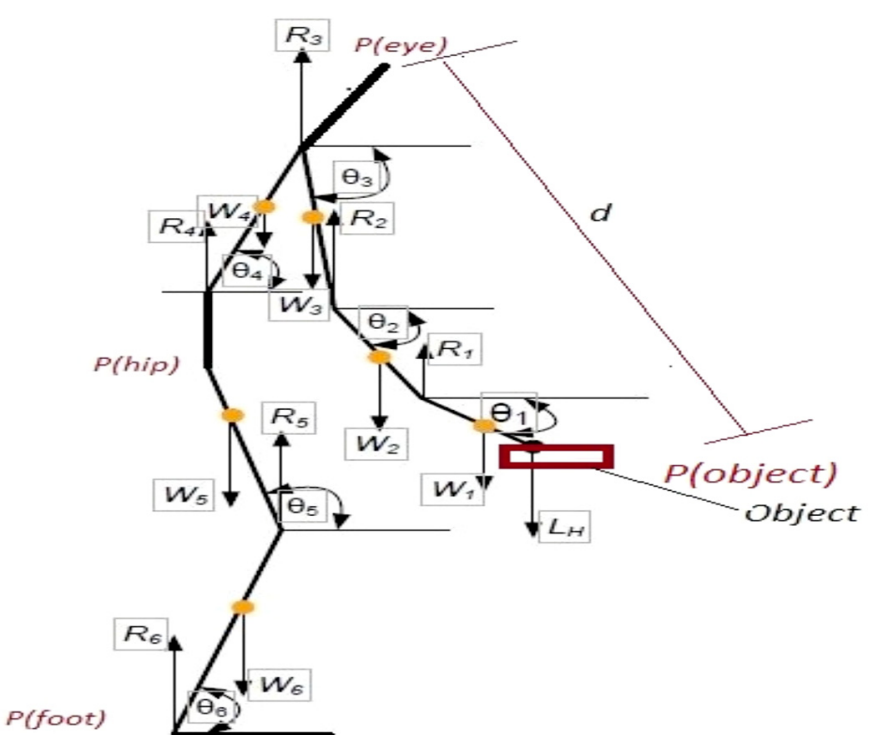

Fig. 2. Model with angulations and visual demand.

\section{Procedure}

Posture prediction of manual assembly is highly required in human models. The predicted posture required for a particular task has to satisfy a large number of constraints due to the task condition. In manual assembly visual demand has a great impact. Visual demand gives clear picture of location of the task to secure the assembly efficiency and the quality. Here in this work same object is to be lifted by two subjects having weight $75 \mathrm{~kg}$ and $58 \mathrm{~kg}$, respectively, taken for assembly operations. Here we have taken a load of $3 \mathrm{~kg}$ to be lifted by two subjects to do a particular task.

The starting point and target points are same for both the subjects as the height and length of the body parts are different for both the subjects. The objective cost functions described in previous section gives rise to different postures which is under consideration in this work.

Figure 2 gives the midsagittal plane along with angulations. As there is no distance between hand and object, $\mathrm{P}$ (hand) is considered to be the object point. The distance between $\mathrm{P}$ (eye) and object is known as visual demand (d). When the point $\mathrm{P}$ (hip) is found out the virtual demand is obtained by inclination $\mathrm{q}_{4}$. In this case $\mathrm{P}$ (hip) controls $\mathrm{q}_{1}, \mathrm{q}_{2}, \mathrm{q}_{3}$ and $\mathrm{q}_{4}$.

\section{Results and discussion}

To test and analyze the procedures described in previous section the different path followed by the operator considered in Figure 3 shows initial position as point 1 and the final point as point 7 . The work piece is to be lifted from point 1 and be kept to point 7 . For this purpose, we have taken three paths. The first path is specified by points $1-2-3-4-5-6-7$, which is the longest path, where 1-2-3-4 is the vertical path and points 4-5-6-7 represents horizontal path. The second path is specified by 1-12-13-14-15-7, which is the shortest path. Path three is the in between path of both path-1 and path-2. Path three is specified by 1-8-9-10-11-7.

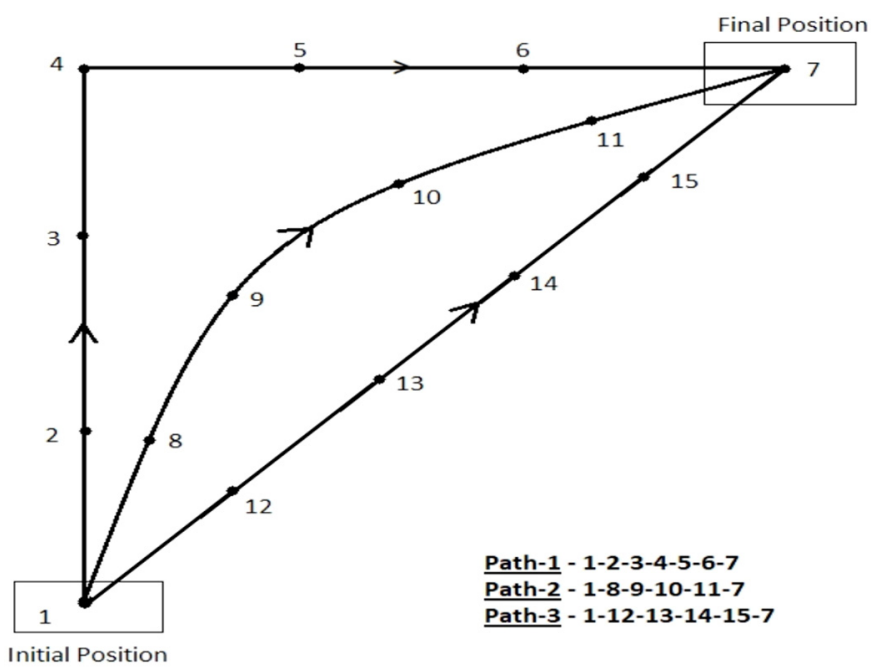

Fig. 3. Paths of operation.

At first the posture prediction is used with optimized discomfort function in all three paths. Discomfort function in all the three paths is shown in Figure 4 for both the subjects. In this figure the discomfort value obtained from the objective function are depicted. In Figure 4a, it is described for path-1 for both the subjects. Similarly, in Figure $4 \mathrm{~b}$ and c it is described for path-2 and path-3, respectively. From the results which are presented in Figure $4 \mathrm{a}-\mathrm{c}$ for subject-1 discomfort is higher in path-1 and path- 2 and it is opposite in path-3 i.e. discomfort is higher in subject-2. It is established that the discomfort is higher in initial conditions and it is found to be gradually decreased.

Then the posture prediction is used with optimized energy expenditure function in all three paths. In Figure 5 energy functions for three paths are shown for both the subjects. In this figure the energy expenditure value obtained from the objective function are depicted. In Figure 5a it is described for path-1 for both the subjects. Similarly, in Figure 5b and c it is described for path- 2 and path-3, respectively. From the results in Figure $5 \mathrm{a}-\mathrm{c}$, it is evident that higher level of energy expenditure is in subject-1 as compared with subject-2. As far as energy expenditure is concerned it is dependent on hip angle. If hip angle i.e. $\Theta_{4}$ increases, energy expenditure decreases and if, $\Theta_{4}$ decreases energy expenditure decreases, so energy expenditure is directly proportional to hip angle.

In above figures discomfort function and energy expenditure for both subjects in different paths are represented. For subject-1 discomfort functions, energy function and the multi objective is shown for all the three paths in Figure 6.

Figure $6 \mathrm{a}-\mathrm{c}$ gives the discomfort function, energy expenditure function and multi objective function for subject-1 for three different paths i.e. path-1, path-2 and path-3. Figure $7 \mathrm{a}-\mathrm{c}$ gives the discomfort function, energy expenditure function and multi objective function for subject-2 for three different paths i.e. path-1, path-2 and path-3. From above figures normalized graphs of objective cost functions, it is found that when discomfort function is considered the curve lies in the top of each figure, while for energy expenditure function, the curve lies on the bottom 


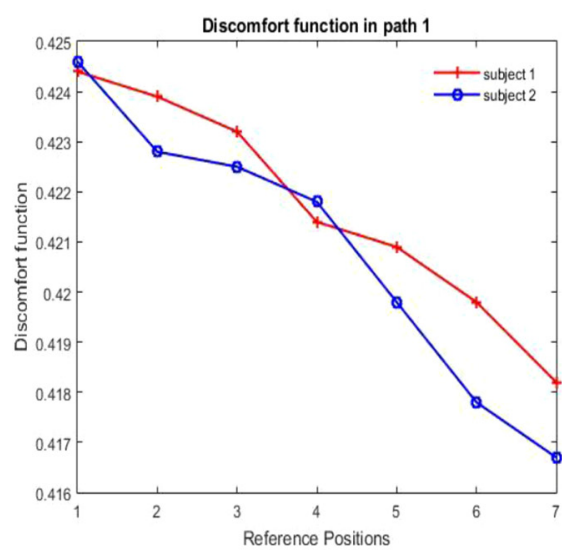

(a)

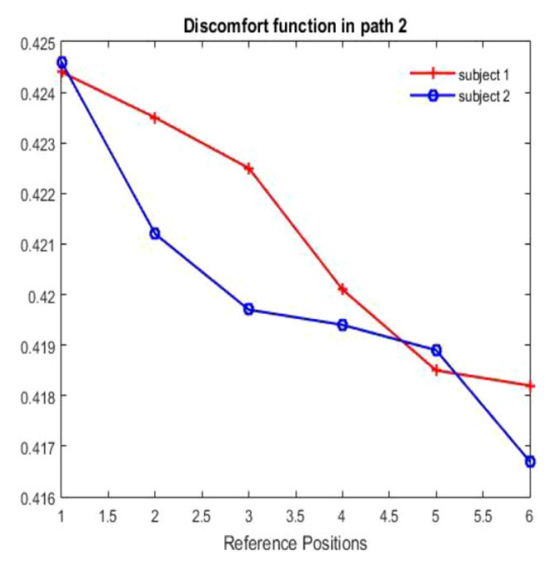

(b)

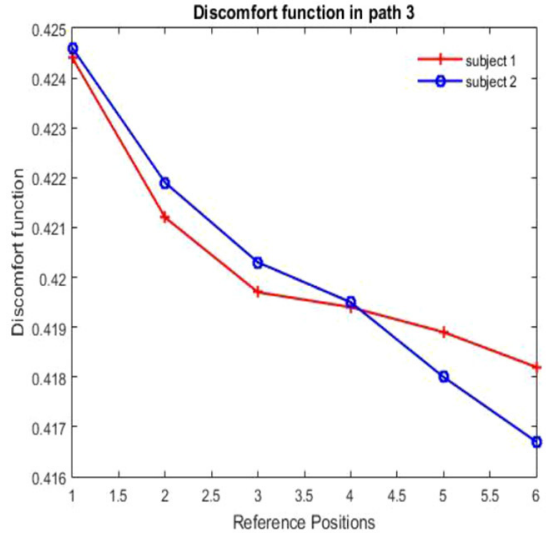

(c)

Fig. 4. Discomfort function in 3 paths.

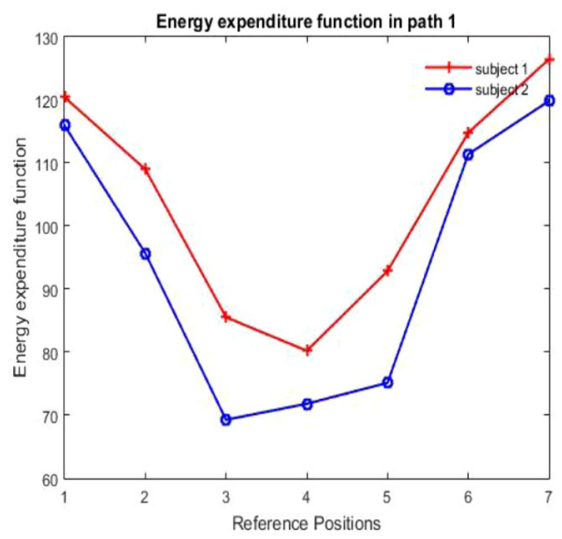

(a)

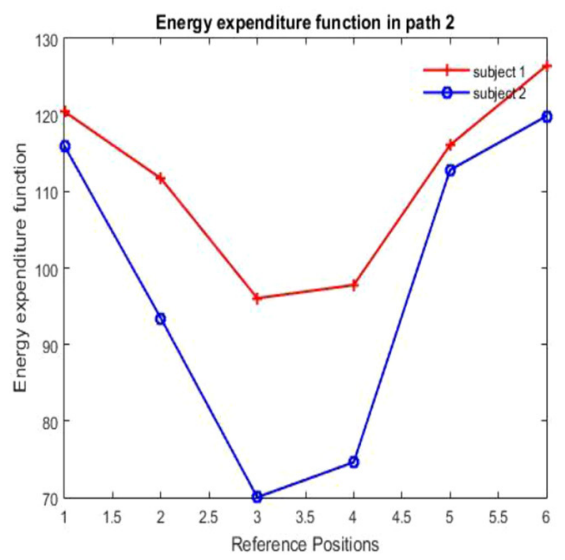

(b)

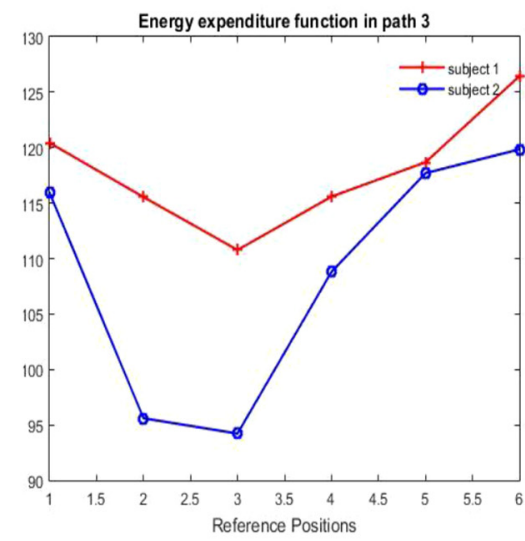

(c)

Fig. 5. Energy expenditure in 3 paths.

of graph. When multi objective is considered the curve lies in between discomfort and energy function. So, in optimization of discomfort, we neglected the total energy required which implies the energy consumption is high. Similarly, in energy expenditure optimization curve, energy expenditure is optimized but not the discomfort, and discomfort function is high. In MOO, both the objective functions are considered and the curve lies in between. So, MOO should be used for prediction of posture. And also, it is marked that when discomfort function increases energy expenditure decreases and if discomfort decreases energy expenditure increases. From Figure 8a-c it is evident that for this particular task subject-2 is better as compared to subject-1 that implies a short heighted person is better for this work. These postures are verified by REBA technique. Pareto front is given for both the subjects in Figures 9 and 10.

REBA procedure involves dividing body segments to coding with respect to different planes. Scoring system for muscle activities is determined for different postures. Coupling activity of load carrying capacity is taken into consideration. REBA scoring system is given by Hignett and McAtamney (2000) [31].
In Table 3, REBA score is given for three different paths taken by the operator for the same work. Initial point score is same for all the paths and the final point score is also same for all three paths. Scores of intermediate points are different for all. From the above table the REBA scores are in between medium risk for all path points.

\section{Conclusion}

In this work a model with 20 DOF of human model is considered for lifting the load in a workspace within reachable limit of the standing operator. The model is developed keeping the ankle joint as the reference point and assumed to be fixed, and changing the other joints to lift the job from its required position to desired position. The work uses to find out the posture of the test examples with these objective measures separately and also in combination as MOO case. The two subjects (of different weight and height) are considered as test examples. Three procedures are used to find out the optimized posture in both the test cases. It is found that the discomfort function is only the measure of the joints of the forearm part as it is found that the hip position almost remains of the same 


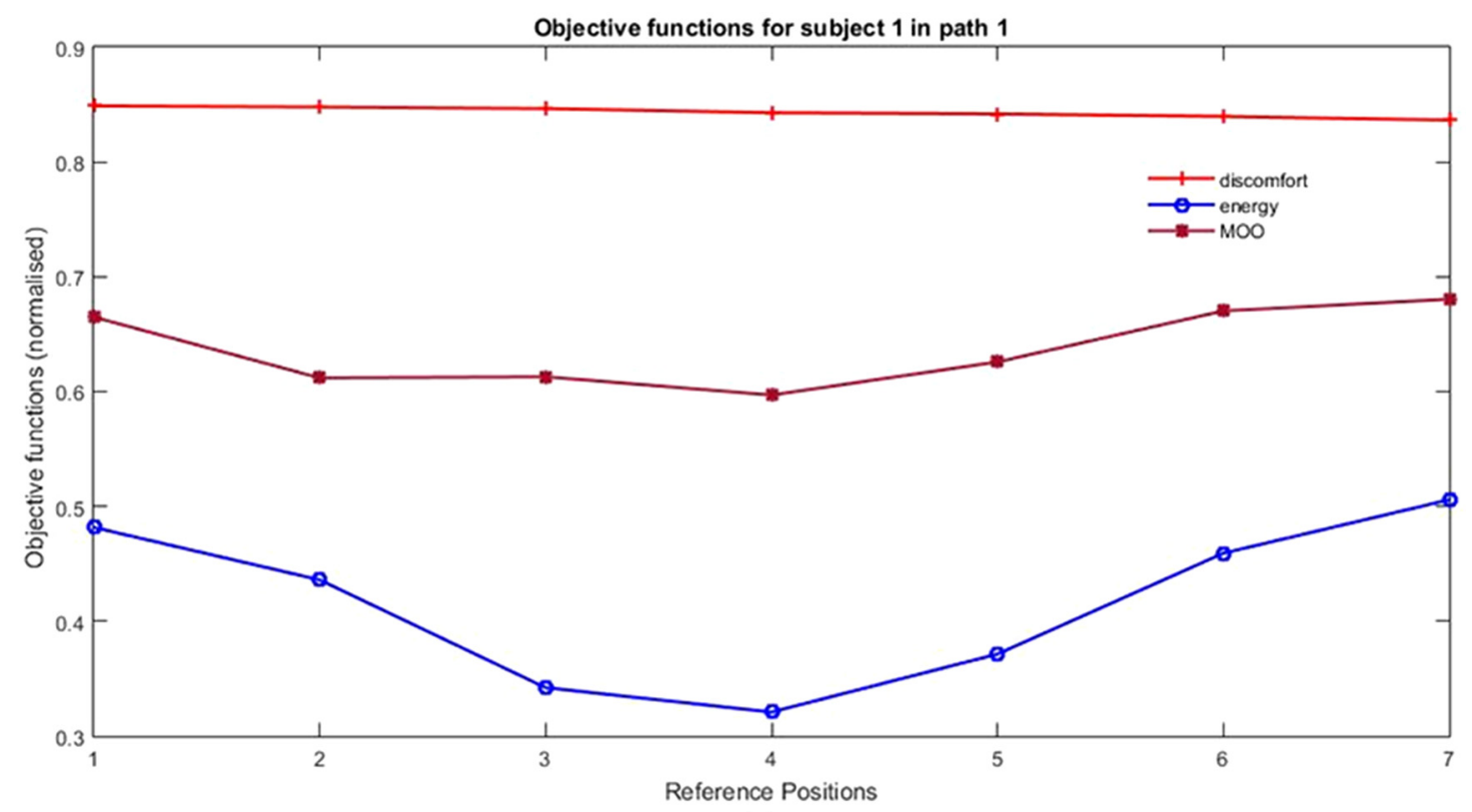

(a)

Objective functions for subject 1 in path 2

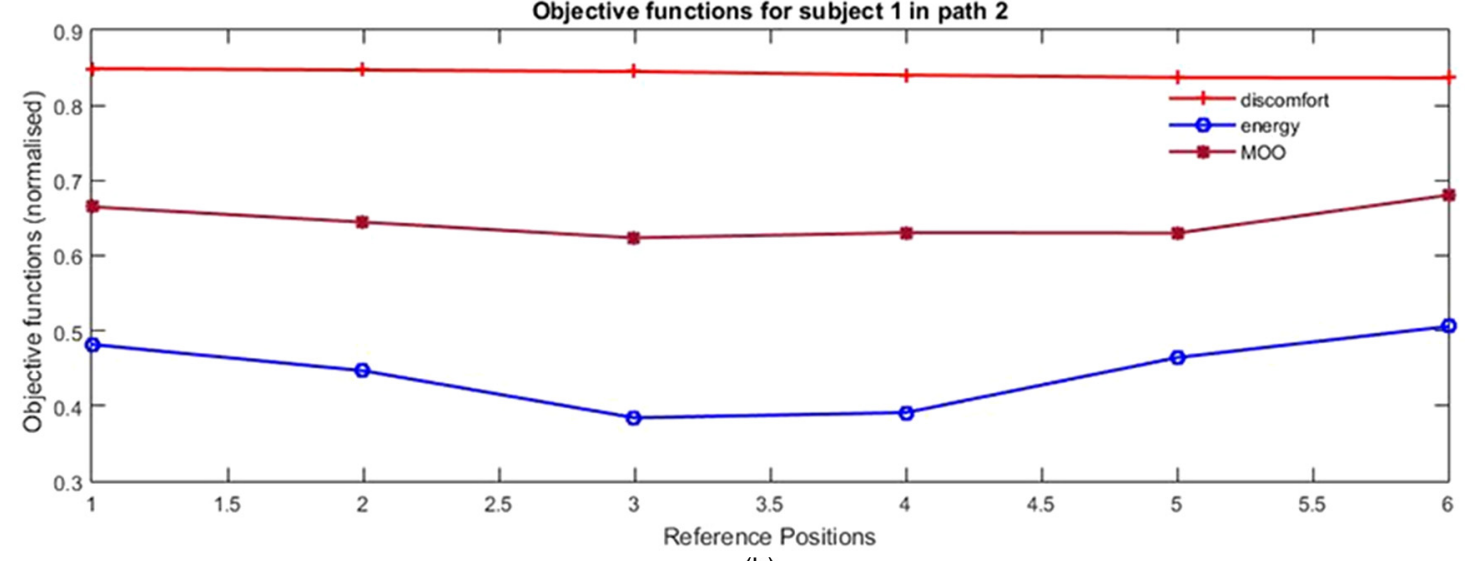

(b)

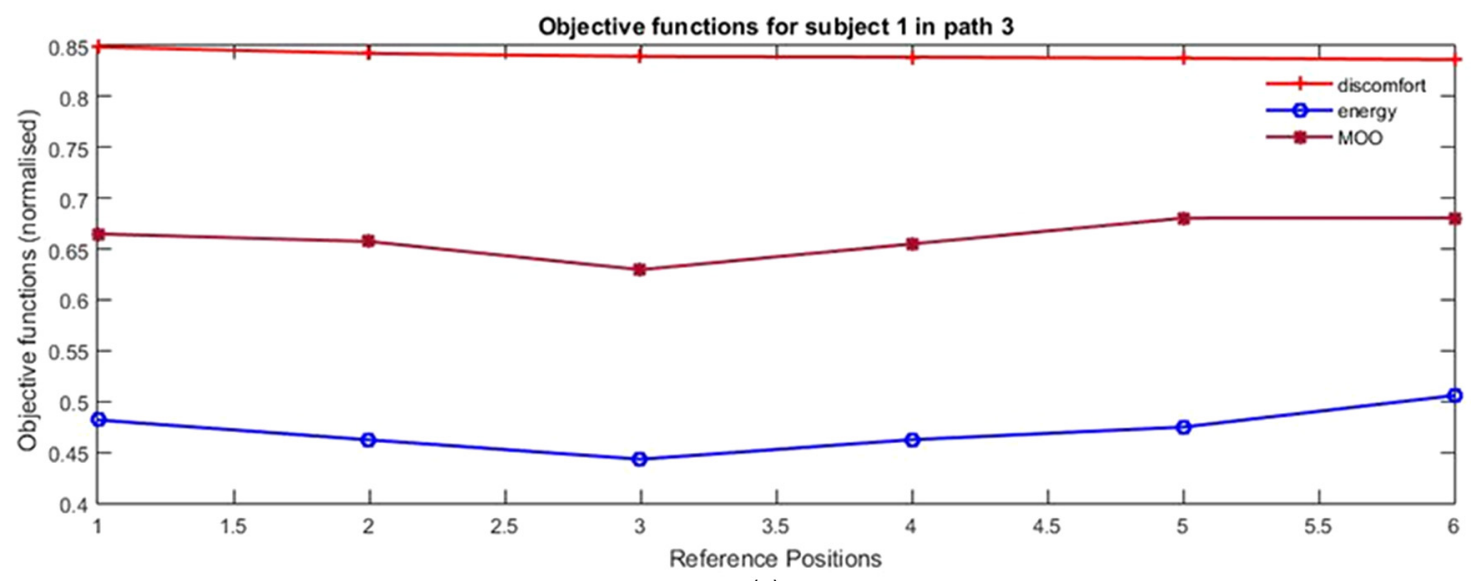

(c)

Fig. 6. Multi objective for subject-1 in path-1 (a), path-2 (b), path-3 (c). 


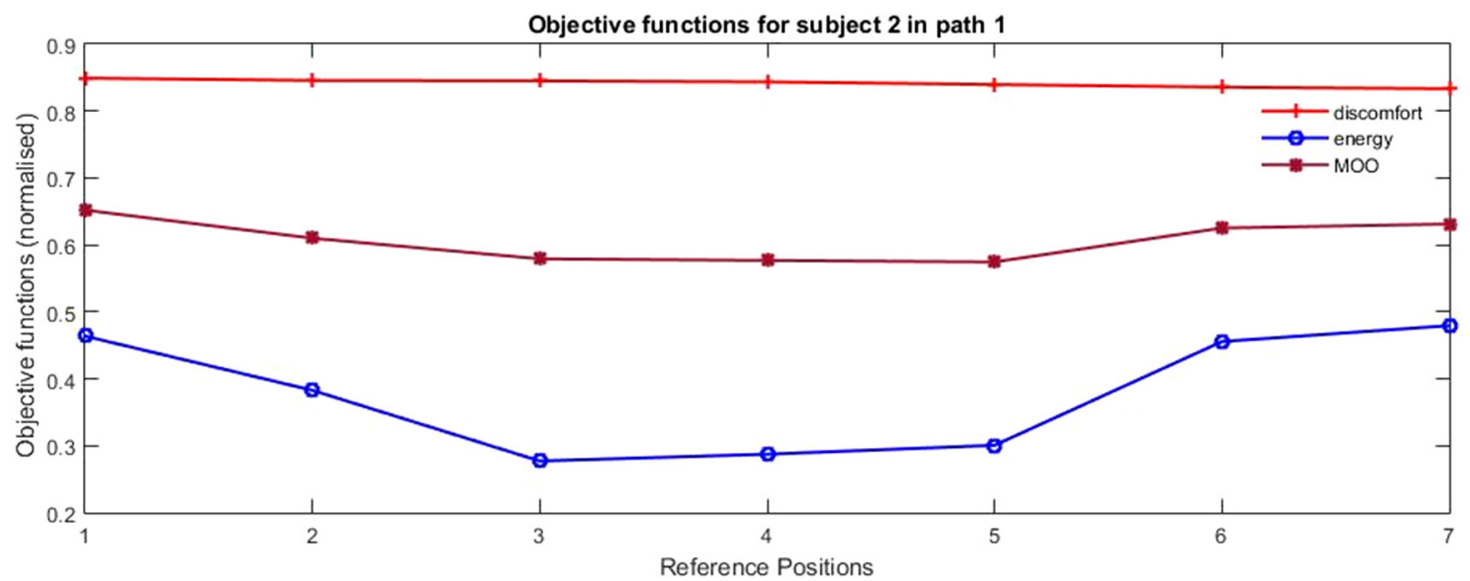

(a)

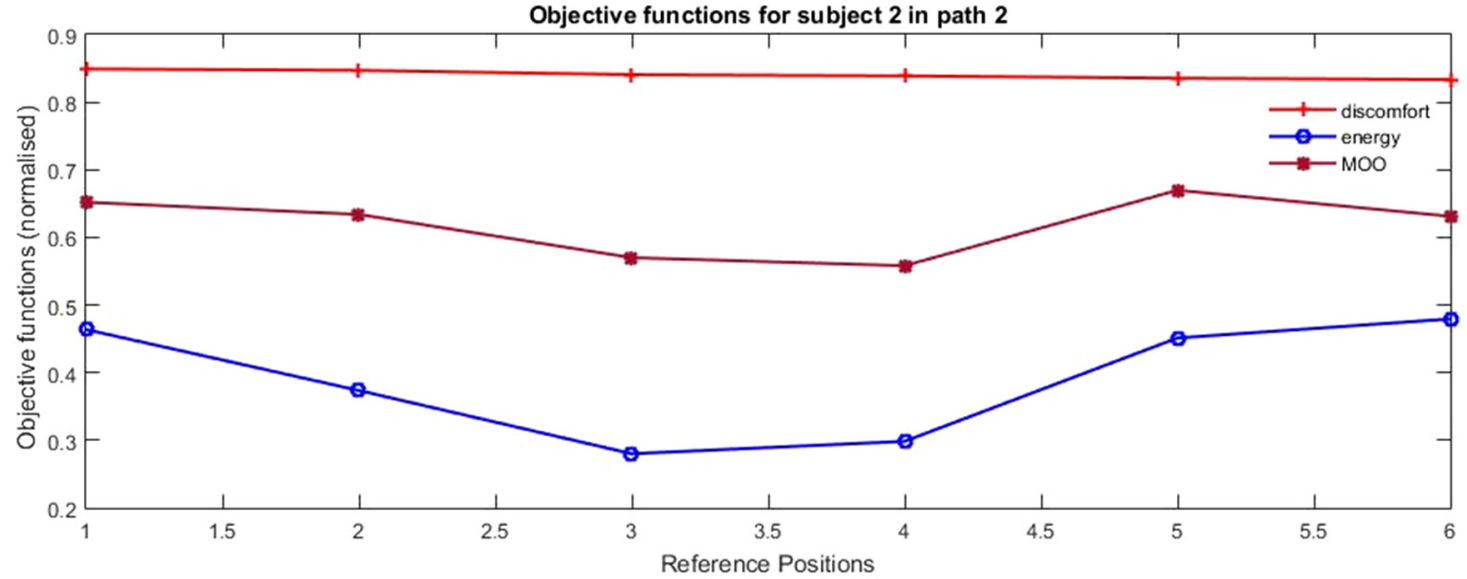

(b)

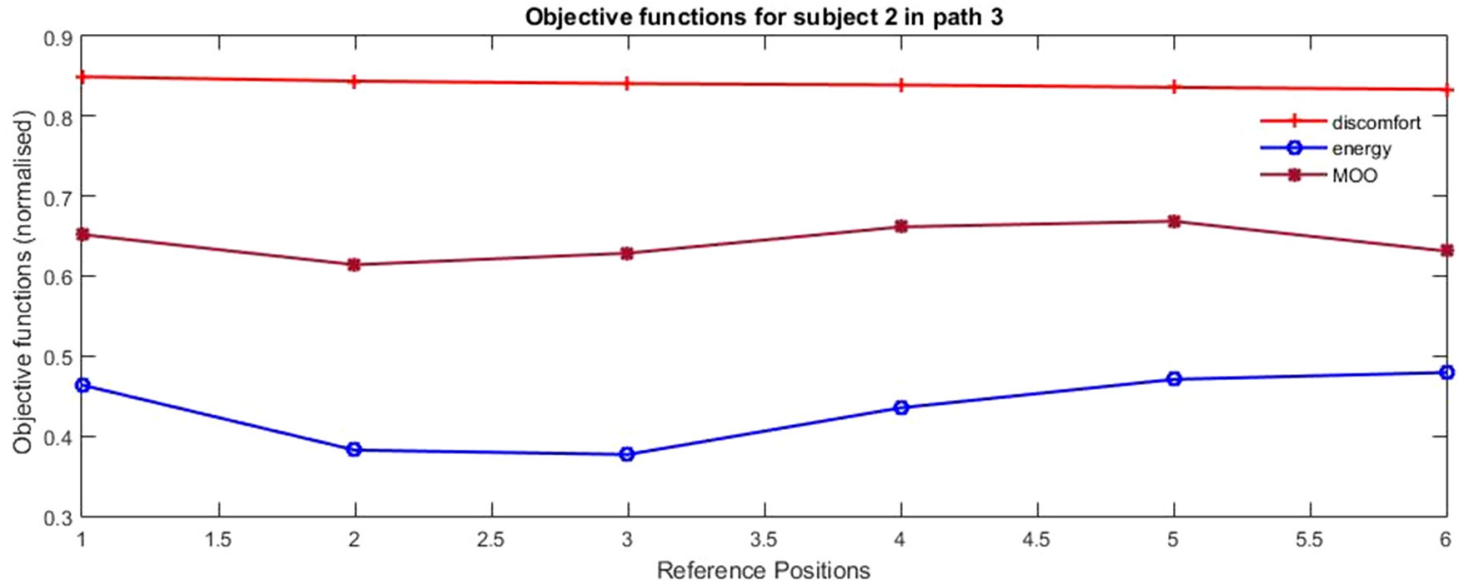

(c)

Fig. 7. Multi objective for subject-2 in path-1 (a), path-2 (b), path-3 (c).

height. In minimum energy expenditure rate hip is at a lower position and using MOO the hip remains in between the both previous parts. The change in joint parameter is such that discomfort at the joint is less and with less energy expenditure which also depicts that the movement is under medium risk in the REBA scale.

Based on the extensive experimental work the following outcomes can be drawn. A short heighted person is better for this operation. The multi objective is a better procedure for posture prediction. When discomfort function increases energy expenditure decreases and if discomfort decreases energy expenditure increases. This paper can be helpful in managing the operators in manual assembly operations in various workplaces. In the extended work a greater number of cost functions can be taken for analysis in different work places. 


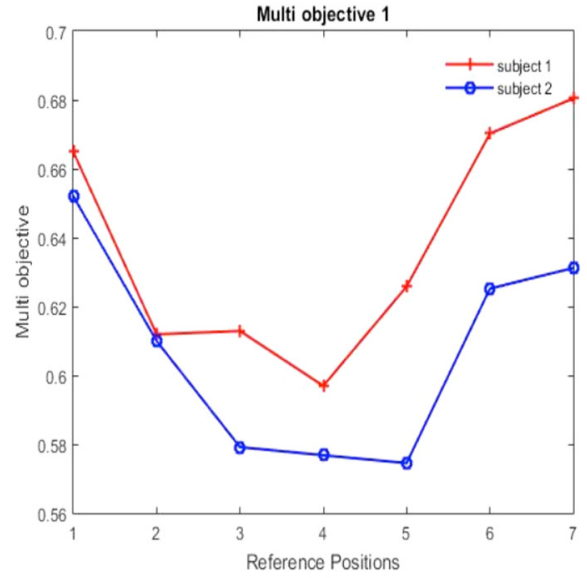

(a)

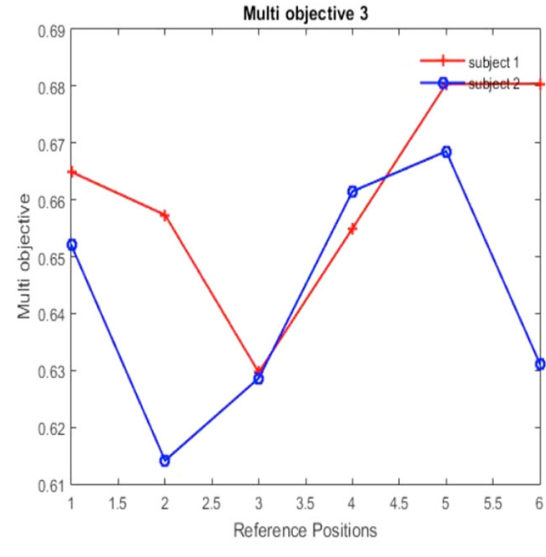

(b)

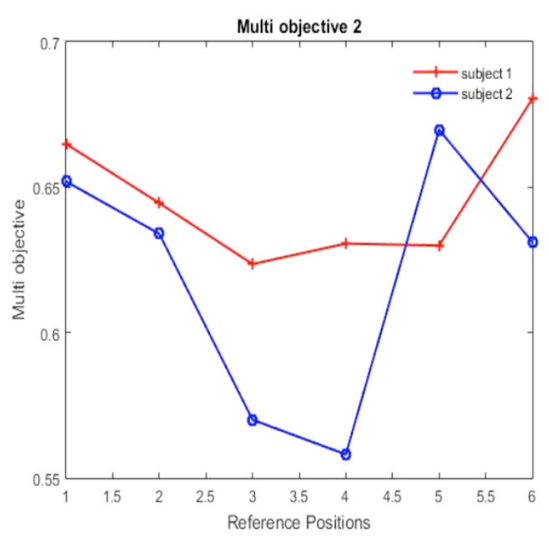

(c)

Fig. 8. Multi objective for path-1 (a), path-2 (b) and path-3 (c).

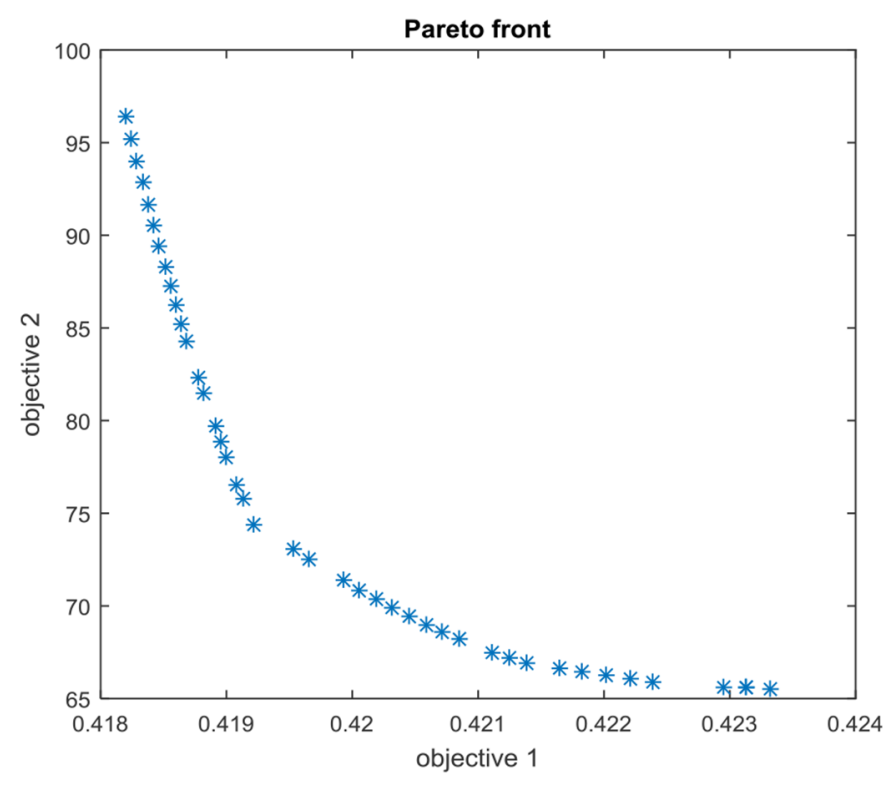

Fig. 9. Pareto front for Subject-1.

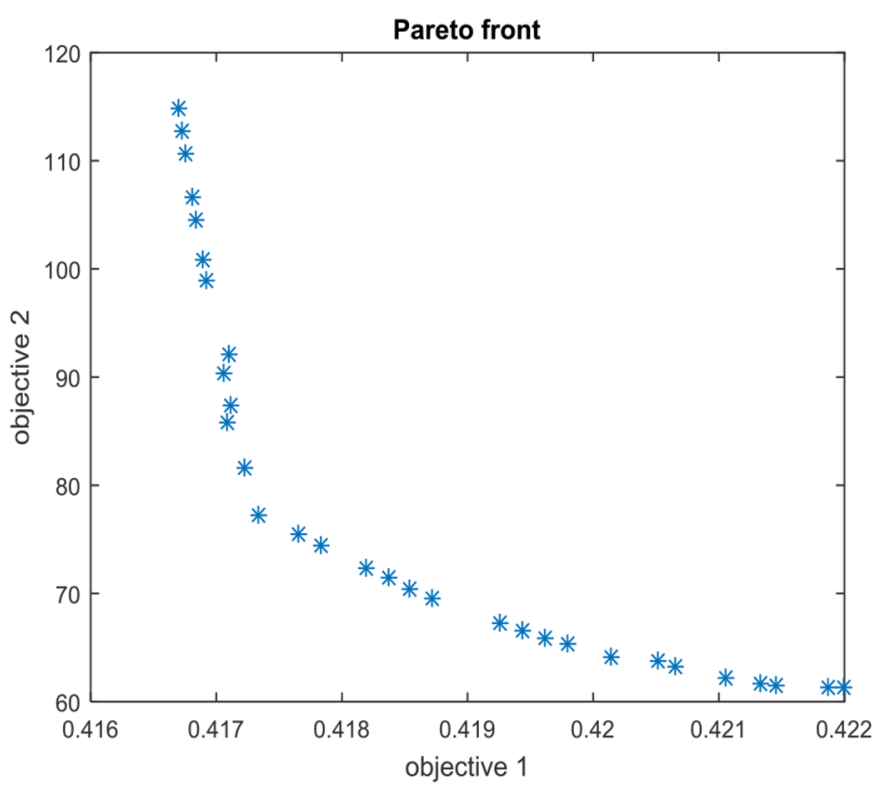

Fig. 10. Pareto front for Subject-2.

Table 3. REBA score in different path. $1=$ Negligible risk; $2-3=$ Low risk, change may be needed; $4-6=$ Medium risk, further investigation, change soon; $7-10=$ High risk, investigate and implement change; $11+=$ Very high risk, change immediately.

\begin{tabular}{llllllll}
\hline & & $\begin{array}{l}\text { Initial } \\
\text { point }\end{array}$ & $\begin{array}{l}\text { Intermediate } \\
\text { point-1 }\end{array}$ & $\begin{array}{l}\text { Intermediate } \\
\text { point-2 }\end{array}$ & Intermediate point-3 & $\begin{array}{l}\text { Intermediate } \\
\text { point-4 }\end{array}$ & $\begin{array}{l}\text { Final } \\
\text { point }\end{array}$ \\
\hline \multirow{4}{*}{ REBA Score } & Path-1 & 5 & 4 & 6 & 6 & 6 & 6 \\
& Path-2 & 5 & 4 & 5 & 5 & 6 & 6 \\
& Path-3 & 5 & 4 & 6 & 5 & 5 & 6 \\
\hline
\end{tabular}




\section{References}

1. B. Rout, P.P. Tripathy, R.R. Dash, D. Dhupal, Optimization of posture prediction using MOO in brick stacking operation, in Computational Intelligence in Data Mining (Springer Science and Business Media LLC, Cham, 2020)

2. B. Rout et al., Optimal posture prediction in brick stacking operation for reducing ergonomic risk factor, Int. J. Recent Technol. Eng. 8, 1835-1841 (2019)

3. V. Lippi, Prediction in the context of a human-inspired posture control model, Robot. Auton. Syst. 107, 63-70 (2018)

4. S.D. Farahani et al., Human arm posture prediction in response to isometric endpoint forces, J. Biomech. 48, 4178$4184(2015)$

5. J.H. Kim et al., Lifting posture analysis in material handling using virtual human, in Proceedings of International Mechanical Engineering Congress and Exposition IMECE2005, November 5-11, 2005, Orlando, Florida USA

6. B. Rout et al., Optimization of posture analysis in manual assembly, Int. J. Mech. Prod. Eng. Res. Dev. 8, 751-764 (2018)

7. A.M. Karim, Y. Jingzhou, M. Timothy, B. Steven, M. Anith, Z. Xianlian, P. Amos, A. Jasbir. Towards a new generation of virtual humans, Int. J. Human Factors Model. Simul. 1, 2-39 (2006)

8. J. Yang, T. Sinokrot, M.K. Abdel, A general analytic approach for santosa upper extremity workspace, Comput. Ind. Eng. 54, 242-258 (2008)

9. B. Rout et al., Effective work procedure design using discomfort and effort factor in brick stacking operation - a case study, IOP Conf. Ser. Mater. Sci. Eng. 310, 012020 (2018)

10. A.K. John, K. Krishnakumar, Performing multiobjective optimization on perforated plate matrix heat exchanger surfaces using genetic algorithm, Int. J. Simul. Multisci. Des. Optim. 8, A3 (2017)

11. M. Chandramouli, G.R. Bertoline, A genetic algorithm based optimization framework to visualize, evaluate, and modify 3D space configurations in Desktop VR, Int. J. Simul. Multisci. Des. Optim. 5, A01 (2014)

12. S. Pheasant, C.M. Haslegrave, Bodyspace: Anthropometry, Ergonomics, and the Design of Work (Taylor and Francis, Boca Raton, FL, 2006)

13. K. Hase, N. Yamazaki, Development of three-dimensional whole-body musculoskeletal model for various motion analyses, JSME Int. J. Ser. C 40, 25-32 (1997)

14. D.B. Chaffin, Improving digital human modelling for proactive ergonomics in design, Ergonomics 48, 478-491 (2005)

15. A. Gholipour, N. Arjmand, Artificial neural networks to predict 3D spinal posture in reaching and lifting activities: applications in biomechanical models, J. Biomech. 49, 29462952 (2016)
16. R.T. Marler, J.S. Arora, J. Yang, H.-J. Kim, K. Abdel-Malek, Use of multi-objective optimization for digital human posture prediction, Eng. Optim. 41, 925-943 (2009)

17. L. Ma, W. Zhang, D. Chablat, F. Bennis, F. Guillaume, Multiobjective optimisation method for posture prediction and analysis with consideration of fatigue effect and its application case, Comput. Ind. Eng. 57, 1235-1246 (2009)

18. R.T. Marler, J.S. Arora, Survey of multi-objective optimization methods for engineering, Struct. Multidiscip. Optim. 26, 369-395 (2004)

19. Y. Xiang et al., Human lifting simulation using a multiobjective optimization approach, Multibody Syst. Dyn. 23, 431-451 (2010)

20. Z. Mi, J. (James) Yang, K. Abdel-Malek, Optimization-based posture prediction for human upper body, Robotica 27, 607620 (2009)

21. E.N. Horn, Optimization-based dynamic human motion prediction, Master Thesis, University of Iowa, 2005

22. F.C. Anderson, M.G. Pandy, Static and dynamic optimization solutions for gait are practically equivalent, J. Biomech. 34, 153-161 (2001)

23. L. Ma, W. Zhang, D. Chablat, F. Bennis, F. Guillaume, Multi objective optimisation method for posture prediction and analysis with consideration of fatigue effect and its application case, Comput. Ind. Eng. 57, 1235-1246(2009)

24. J. Denavit, R.S. Hartenberg, A kinematic notation for lowerpair mechanisms based on matrices, ASME J. Appl. Mech. 23, 215-221 (1955)

25. R.T. Marler, S. Rahmatalla, M. Shanahan, K. Abdel-Malek, A new discomfort function for optimization-based posture prediction, in Proceedings of the SAE Human Modeling for Design and Engineering Conference, 2005, p. 2680

26. Q.L. Zou, Q.H. Zhang, J.Z. Yang, J. Gragg, An inverse optimization approach for determining weights of joint displacement objective function for upper body kinematic posture prediction, Robotica 30, 389-404 (2012)

27. B.R. Umberger, K.G. Gerritsen, P.E. Martin, A model of human muscle energy expenditure, Comput. Methods Biomech. Biomed. Eng. 6, 99-111 (2003)

28. J.H. Kim, K. Abdel-Malek, J. Yang, T. Marler, K. Nebel, Lifting posture analysis in material handling using virtual humans, Proc. ASME Int. Conf. Manuf. Sci. Eng. 16, 14451453 (2005)

29. K. Hase, N. Yamazaki, Development of three-dimensional whole-body musculoskeletal model for various motion analyses, JSME Int. J. Ser. C Dyn. Control Robot. Des. Manuf. 40, 25-32 (1997)

30. L. Ma, W. Zhang, D. Chablat, F. Bennis, F. Guillaume, Multiobjective optimisation method for posture prediction and analysis with consideration of fatigue effect and its application case, Comput. Ind. Eng. 57, 1235-1246 (2009)

31. S. Hignett, L. McAtamney, Rapid Entire Body Assessment (REBA), Appl. Ergon. 31, 201-205 (2000)

Cite this article as: Biswaranjan Rout, Rati Ranjan Dash, Debabrata Dhupal, Posture prediction and optimization for a manual assembly operation involving lifting of weights, Int. J. Simul. Multidisci. Des. Optim. 11, 1 (2020) 